International Mathematical Forum, 1, 2006, no. 38, 1887-1892

\title{
The Left Invariant Metrics which is Defined on Heisenberg Group
}

\author{
Essin TURHAN and Necdet CATALBAS \\ Firat University, Department of Mathematics \\ 2119,Elazı̆̆, Turkey \\ eturhan@firat.edu.tr
}

\begin{abstract}
In this paper it is computed some curvatures by means of the left invariant metrics. An important role is played by the Heisenberg manifold.The curvatures are calculated from various vector fields.

Mathematics Subject Classification: Principal: 37J60; Secondary: 53B21, 70H03
\end{abstract}

Keywords: Ricci, Riemann, curvature,geodesics, Left invariant metric

\section{I.Introduction}

Riemannian geometry and elliptic operators are closely related. The most simple Riemannian metric is the flat one and the associated elliptic operator is the usual laplacian which is invariant under translations on the additive group $(\mathrm{IR},+)$. In an analogus way, for the sub-Riemanian geometry, the associated operator is sub-elliptic(see $\left[\mathrm{BGG}_{1}\right],\left[\mathrm{BGG}_{2}\right]$ ). Heisenberg group is the prototype for this geometry. It is the simplest non-commutative Lie group.

An important application of the Heisenberg group is the non-commutative geometric analysis approach to clinical MRI.(see Sch). This is based on the temporal magnetic resonance phenomeon discovered by Bloch(see $(\mathrm{Fu}))$. He detected an induction field of nuclear orijin, which indicates a rotation of the total oscillating field around a constant magnetics field.The static magnetic field is described by the missing direction $\partial_{z}$. This is given by the Lie bracket of the vector fields $X_{1}$ and $X_{2}$. The nuclear field acts at resonance over the larmaur orbits. The Larmour equation for the magnetic spin precession describes the geodesics on the Heisenberg group.The vector fields $X_{1}, X_{2}$ and $\partial_{z}$ span the Lie algebra of the Heisenberg group.

Now, consider the following group law on $\mathrm{IR}^{3}$ 


$$
(x, y, z) \circ(a, b, c)=(x+a, y+b, z+c) .
$$

The pair $\left(I R^{3}, \circ\right)$ is a Lie group denoted by $H^{1}$. The vector fields

$$
X_{1}=\partial x+2 x \partial z, X_{2}=\partial y+\partial x \partial z, T=\partial z
$$

span the Lie algebra of the Heisenberg group $H^{1}$. It is easily seen that

$$
w\left(X_{1}\right)=w\left(X_{2}\right)=0, w(T)=1
$$

and

$$
\left[X_{1}, X_{2}\right]=-4 T
$$

Also,the following relations are valid

$$
h\left(X_{i}, X_{j}\right)=\delta_{i j}, h\left(X_{i}, T\right)=0, h(T, T)=\lambda
$$

where $h$ define a unique Riemannian metric.According to these relations,the coefficients are given by

$$
h_{i j}^{(\lambda)}=\left[\begin{array}{ccc}
1+4 \lambda y^{2} & -4 \lambda x y & -2 \lambda y \\
-4 \lambda x y & 1+4 \lambda x^{2} & 2 \lambda x \\
-2 \lambda y & 2 \lambda x & \lambda
\end{array}\right]
$$

Now, we give the following Lemma:

Lemma I.1. If $R_{i j}^{(\lambda)}$ are the components of the Ricci tensor with respect to the metric $h_{i j}^{(\lambda)}$ on $H^{1}$, then $R_{i j}^{(\lambda)}=-8 \lambda h_{i j}^{(-\lambda)}$.

Proposition I.1. For any vector field $V$ we have

$$
R^{(\lambda)}(V, V)=8 \lambda\left(h^{(\lambda)}(V, V)-2 \lambda w(V)^{2}\right) .
$$

\section{General Case}

We have investigated the case when the vector fields are given by the formula (1.2). In this section we will dal with the more general case of the vector fields, that is,

$$
X_{1}=\partial x+A_{1}(y) \partial z, X_{2}=\partial y-A_{2}(x) \partial z
$$


with $A_{i}($.$) are continuous functions. In this case the 1$-form can be written in the following form :

$$
w=d z-A_{1}(y) d x+A_{2}(x) d y .
$$

When considering these vector fields, Riemann metrics serve to establish the following matrix :

$$
h_{i j}^{(\lambda)}=\left[\begin{array}{ccc}
1+\lambda A_{1}^{2}(y) & -\lambda A_{1}(y) A_{2}(x) & -\lambda A_{1}(y) \\
-\lambda A_{1}(y) A_{2}(x) & 1+\lambda A_{1}^{2}(y) & \lambda A_{2}(x) \\
-\lambda A_{1}(y) & \lambda A_{2}(x) & \lambda
\end{array}\right]
$$

Lemma II.1. If $R_{i j}^{(\lambda)}$ are the components of the Ricci tensör with respect to the metrics $h_{i j}^{(\lambda)}$ on $H^{1}$, then

$$
R_{i j}^{(\lambda)}=R_{i j}^{(-\lambda)}+\frac{\lambda}{2} Q_{i j}
$$

where $h_{i j}$ computed by mean of Lemma I.1,

$$
Q_{i j}=\left[\begin{array}{ccc}
2 A_{1} A_{1}^{\prime \prime} & -\left(A_{1}^{\prime \prime} A_{2}+A_{1} A_{2}^{\prime \prime}\right) & -A_{1}^{\prime \prime} \\
-\left(A_{1}^{\prime \prime} A_{2}+A_{1} A_{2}^{\prime \prime}\right) & 2 A_{2} A_{2}^{\prime \prime} & A_{2}^{\prime \prime} \\
-A_{1}^{\prime \prime} & A_{2}^{\prime \prime} & 0
\end{array}\right]
$$

and $R$ is the Ricci scalar [1].

III.The curvature on the Heisenberg group which is computed via left invariant metric

We call the Lie group generalized Heisenberg group which constitutes the matrices of following form

$$
\left[\begin{array}{ccc}
I_{r} & P & T \\
0 & 1 & Q \\
0 & 0 & 1
\end{array}\right], r \geq 1
$$

where $\mathrm{P}$ and $\mathrm{T}$,are the column matrices and $Q \in I R$. It is represented by $H(1 . r)$.

The $H(1, r)$ Lie group is diffeomorfic to $I R^{2 r+1}$,that is the transformation

$$
\begin{gathered}
\Psi: I R^{2 r+1} \rightarrow H(1 . r) \\
\Psi(X, Y, Z)=\left[\begin{array}{ccc}
I_{R} & -Z^{t} & X \\
0 & 1 & Y \\
0 & 0 & 1
\end{array}\right]
\end{gathered}
$$


defines a diffeomorphism where $X \in I R_{m}^{r}, Z \in I R_{1}^{m} \quad$ [2].

Let us denote the Lie group of the affin transformations of $I R^{2 r+1}$ with $A(2 r+1, I R)$. The set of the matrices with the following from is the subset of the Lie group

$$
A(2 r+1): G_{0}(1, r)=\left[\begin{array}{cccc}
I_{R} & -D^{t} & 0 & B \\
0 & 1 & 0 & C \\
0 & 0 & 1 & H
\end{array}\right], H \in I R^{n}
$$

This subset can be made isomorphism to generalized Heisenberg Lie group $H(1, r)$. That is ,the transformation

$$
\begin{gathered}
\Phi: G_{0}(1, r) \rightarrow H(1, r) \\
(B, C, D) \rightarrow\left[\begin{array}{ccc}
I_{R} & -D^{t} & B \\
0 & 1 & C \\
0 & 0 & 1
\end{array}\right]
\end{gathered}
$$

defines a Lie group isomorphism of $G_{0}(1, r)$ to the generator $(B, C, D)$.Now, if we choose $D, B \in Z^{m}$ and $C \in Z$, then we get a Lie subgroup of $G(1, n)$.Let us denote this Lie subgroup with $\Gamma_{0}, \Phi\left(\Gamma_{0}\right)$ the subgroup of $H(1, r)$ which the matrices are integers with $\Gamma$. Hence we denote the manifold $H(1, r) / \Gamma$ which is obtained in this way briefly by $M^{2 r+1}$. This manifold is compact and complete.Furthermore, its fundamental group is isomorphic to $\Gamma$ [2]. Where $\Gamma$ ,is a subgroup of $A(2 r+2)$. Now we calculate the curvatures on this manifold. In oder to do this we consider the set $\mathrm{G}$,which its elements constitutes with the following type of matrices.

$$
\left[\begin{array}{ccccccc}
1 & 0 & \cdots & 0 & a_{1 r+1} & a_{1 r+2} & 0 \\
0 & 1 & \cdots & 0 & a_{2 r+1} & a_{2 r+1} & 0 \\
\cdots & \cdots & \cdots & \cdots & \cdots & \cdots & \cdots \\
0 & 0 & \cdots & 1 & a_{r r+1} & a_{r r+2} & 0 \\
0 & 0 & \cdots & 0 & 1 & a_{r+1 r+2} & 0 \\
0 & 0 & 0 & 0 & 0 & 1 & 0 \\
0 & 0 & 0 & 0 & 0 & 0 & e^{2 \pi a i}
\end{array}\right], a \in I R .
$$

It is well known that $G=H(1, r) \times S^{1}$ and $G$ is diffeomorphic to the $G / \Gamma$ group [2].

Let the coordinat functions on the $G$ for $11 \leq i \leq$ be $X_{i}, Y, Z_{i}, T$. For $A \in G$, we define these functions with the following

$$
X(A)=a_{i}^{r+1}, Y(A)=a_{r+1}^{r+i}, \quad Z_{i}(A)=a_{i}^{r+2}, T(A)=a .
$$


Let us define the new matrix that (i) denotes the $(n \times 1)$-column matrix with 1 at the ith row:

$$
\alpha_{i}=\left[\begin{array}{ccc}
I_{R} & (i) & 0 \\
0 & 1 & 0 \\
0 & 0 & 1
\end{array}\right], \beta=\left[\begin{array}{ccc}
I_{R} & 0 & 0 \\
0 & 1 & 1 \\
0 & 0 & 1
\end{array}\right], \gamma_{i}=\left[\begin{array}{ccc}
I_{R} & 0 & (i) \\
0 & 1 & 1 \\
0 & 0 & 1
\end{array}\right]
$$

They generates the noncommutative $\Gamma$ free group.Using these matrices we define linearly independent and left invariant 1-form family:

$$
\alpha_{i}=d x, \beta=d y, \gamma=d z_{i}-x_{i} d y, \eta=d t .
$$

The dual vectors of these can be given respectively in the following form:

$$
X_{i}=\frac{\partial}{\partial x_{i}}, Y=\frac{\partial}{\partial y}+\sum_{i=1}^{r} x_{i} \frac{\partial}{\partial z_{i}}, Z_{i}=\frac{\partial}{\partial z_{i}}, T=\frac{\partial}{\partial t} .
$$

Thus, the let $\left\{X_{i}, Y, Z_{i}, T\right\}$ form an orthonormal vector fields whit respect to the left invariant metric tensor on $\mathrm{G}$ defined

$$
d s^{2}=\sum_{i=1}^{r}\left(\alpha_{i}^{2}+\gamma_{i}^{2}\right)+\beta^{2}+\eta^{2} .
$$

According to this define the Ricci tensor on the manifold $M^{2 r+2}$.

Now let us defined

$r(U, V)=\sum_{i=1}^{r}\left\{R\left(U, X_{i}, V, X_{i}\right)+R\left(U, Z_{i}, V, Z_{i}\right)+R(U, Y, V, Y)+R(U, T, V, T)\right\}$

where $\mathrm{U}$ and $\mathrm{V}$ are tanget vectors of $M^{2 r+2}$ and $\left\{X_{i}, Y, Z_{i}, T\right\}$ are the orthonormal base vector fields of $M^{2 r+2}$.

Then

$$
\begin{aligned}
& \nabla_{X_{i}} Z_{i}=\nabla_{Z_{i}} X_{i}=-\frac{1}{2} Y \\
& \nabla_{X_{i}} Y=\nabla_{Y} X_{i}=-\frac{1}{2} Z_{i} \\
& \nabla_{Z_{i}} Y=\nabla_{Y} Z_{i}=-\frac{1}{2} X_{i}
\end{aligned}
$$

Hence we obtain the following result:

$$
R\left(X_{i}, Z_{i}, X_{i}, Z_{i}\right)=\left\langle R\left(X_{i}, Z_{i}, X_{i}\right), Z i\right\rangle
$$




$$
\begin{gathered}
=\left\langle\nabla_{X_{i}} \nabla_{Z_{i}} X_{i}-\nabla_{Z_{i}} \nabla_{X_{i}} X_{i}-\nabla_{\left[X_{i}, Z_{i}\right]} X_{i}, Z_{i}\right\rangle \\
=\left\langle\nabla_{X_{i}}\left(-\frac{1}{2} Y\right), Z_{i}\right\rangle=-\frac{1}{4}
\end{gathered}
$$

In a similar way, we have

$$
\begin{aligned}
R\left(X_{i}, Y, X_{i}, Y\right) & =\frac{3}{4} \\
R\left(Z_{i}, Y, Z_{i}, Y\right) & =-\frac{1}{4}
\end{aligned}
$$

So,by means of the base $\left\{X_{i}, Y, Z_{i}, T\right\}$, we obtain the ricci tensor in the following form:

$$
\begin{gathered}
r\left(X_{i}, X_{i}\right)=\sum_{i=1}^{r}\left\{R\left(X_{i}, X_{i}, X_{i}, X_{i}\right)+R\left(X_{i}, Z_{i}, X_{i}, Z_{i}\right)\right\} \\
+\sum_{i=1}^{r}\left\{R\left(X_{i}, Y, X_{i}, Y\right)+R\left(X_{i}, T, X_{i}, T\right)\right\} \\
=\sum_{i=1}^{r}\left\{R\left(X_{i}, Z_{i}, X_{i}, Z_{i}\right)+R\left(X_{i}, Y, X_{i}, Y\right)\right\} \\
=-\frac{1}{4}+\frac{3}{4}=\frac{1}{2}
\end{gathered}
$$

Similary, we have $r\left(Z_{i}, Z_{i}\right)=-\frac{1}{2}$ and $r(Y, Y)=\frac{1}{2}$.

Now we are ready to give the following theorem:

Theorem II.1. The scalar curvature of $M$ Lie group,according to the orthonormal base is $\frac{1}{2}$.

Proof: The proof is a matter simply of calculation.

$$
\rho=\sum_{i=1}^{r}\left[r\left(X_{i}, X_{i}\right)+r\left(Z_{i}, Z_{i}\right)\right]+r(Y, Y)+r(T, T)=\frac{1}{2} .
$$

\section{References}

[1] . CALIN,O-MANGIONE,V.(2000) "Geodesics with constraints on Heisenberg manifolds"

[2] HELGASON,S.(1978) "Differential geometry,Lie groups and symmetrics spaces" Newyork ,Acad.Press.

[3] MILNOR,J.(1976) "Curvature of Left Invariant Metrics on Lie Groups" Advances in Math.21,293-329.

[4] MARENICH,V.(1997) "Geodesics in Heisenberg groups" Geom. Dedicata 66,175-185.,1997

Received: February 27, 2006 